\title{
Apollonius diagrams and the Representation of Sounds and Music
}

\author{
Alex McLean \\ Goldsmiths College \\ University of London \\ ma503am@gold.ac.uk
}

\author{
Frederic Fol Leymarie \\ Goldsmiths College \\ University of London \\ ffl@gold.ac.uk
}

\author{
Geraint Wiggins \\ Goldsmiths College \\ University of London \\ geraint.wiggins@gold.ac.uk
}

\begin{abstract}
We introduce the use of weighted medial graph structures to represent sounds and musical events. Such structures permit the building of hierarchical neighborhood relationships (symmetries) while mapping time to a weight (of a note, or event). This is useful to relate perceptual organisations (groupings in the sense of Gestalt theory) both visually and aurally. We treat time in a cinematographic way, effectively using a spacetime representation. The use of colour, modulation (of waveforms), orientation, speed, etc., allows us to integrate multiple musical features in our dynamic diagrams. We provide illustrations, including animations, of our ideas.
\end{abstract}

\section{Introduction}

We seek ways of visually representing the structure and features of sounds and music which are not obvious to distinguish and comprehend, especially to the naive, un-trained, listener. Towards that goal we focus our efforts on medial structures which can support perceptual phenomena such as groupings, (smooth) continuation, and regional or global symmetries, as studied in Gestalt theory [5].

The particular representation of such medial structures we study here takes the form of the weighted Voronoi (or Apollonius) diagram [8] (Fig. 1). The Voronoi diagram is a complete partition of space given a set of events or sites, which in our case will represent notes or sound events. The space selected will span dimensions relevant to aural perception (e.g., pitch or volume). Each Voronoi region (or cell) surrounding an event represents a zone of influence of that event, while Voronoi boundaries are the "symmetries" existing between events by pairs. The weight of a note, e.g., its volume at a given time, can then be used to modify the relative influence of one event with respect to another.

\section{Background}

While Voronoi diagrams represent a truly crossdisciplinary field of research, their application in the area of music has been rather limited. However, a few related applications can be found in the recent literature which either focus on the grouping of notes, the interpolation between musical parameters or the physical modelling of acoustics in space. We summarise these works in this section.

Grouping - Music Polyphony Hamanaka and Hirata described an application of Voronoi diagrams [3] with reference to Lerdahl and Jackendoff's "Generative Theory of Tonal Music" (GTTM) [4]. They consider music as a two dimensional space, with one dimension representing the pitch - the perceived fundamental frequency of a sound - and the other representing time. They use two of the seven Grouping Preference Rules (GPR) of the GTTM; GPR2 and GPR3, where the former is the rule of proximity (in time) and the latter is the rule of change; thus, they relate GPR2 to the time axis and GPR3 to the pitch axis.

They point out a problem of scaling. By mapping time and pitch interval to the Euclidean space of a Voronoi diagram, they mix three different systems of measurements. How much distance is dedicated per time measurement governs how much weight time has over distance, with great influence over the results. This amounts to a conflict between GPR2 and GPR3. This type of conflict is to be expected. The GTTM is a direct application of Gestalt theory to music, and as such contains a number of conflicting (or inter-related) rules. Research is ongoing to quantify the relationships between the rules which cause conflicts. Hamanaka and Hirata's scaling amounts to such a possible quantification.

They also offer a single rule for how groups should be found: "the smallest Voronoi cell is first merged to an adjacent group." They do not go into further detail 


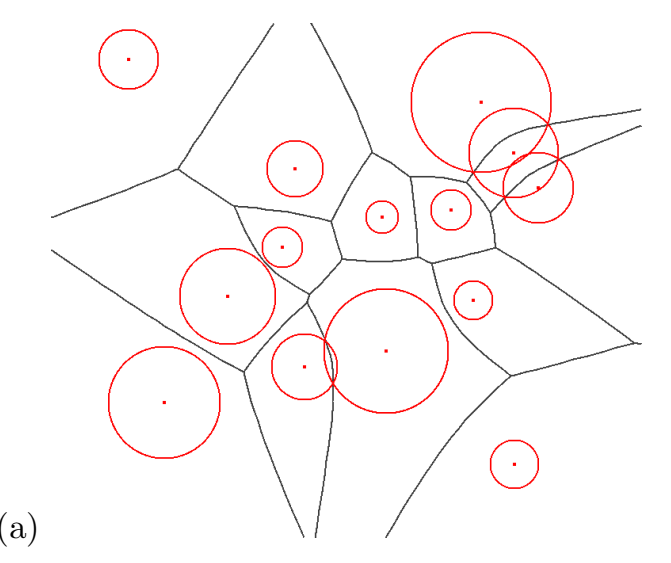

(a)

\section{Figure 1. Apollonius diagram of discs, showing (a) the weighted Voronoi diagram boundaries and (b) its associated (dual) Delaunay tessellation.}

in their paper, leaving some questions open, such as dealing with a lack of adjacency or with multiplicity in adjacency. In summary, they present an interesting approach to the visualisation of the GTTM, but it covers only a small part of this model, leaving room for further work.

An experiment showing a similar approach to mapping but in the area of live music visualisation is introduced in Section 4.1.

The Metasurface Bencina [1] describes a graphical user interface for controlling musical synthesis parameters which he called the metasurface. The user manipulates a Voronoi diagram, where each site represents a particular set of parameters and such that each site may be placed anywhere within the $2 \mathrm{D}$ space. The user then picks a point within that space in order to select each synthesis parameter according to the values of its natural neighbours. The closer neighbours have a higher weighting, so the user may move their pointer across the 2D surface, smoothly interpolating between several sets of parameters at the same time. Here the Voronoi diagram allows a mapping from two to many dimensions, providing a highly intuitive user interface.

Physical modelling synthesis - Waveguide mesh One common method used to simulate the acoustics or reverberation of a room is to model a three dimensional grid of strings stretched across it, with sound waves travelling back and forth along them, scattering where strings cross. These strings are known as waveguides, and this particular technique a waveguide mesh.

However the regularity of this waveguide mesh imposes a kind of city-block rather than Euclidean dis- tance upon the space, resulting in propagation errors and unpleasant distortion. Schiettecatte, Nackaerts and Moor [7] introduce an elegant solution where the edges of a Delaunay tessellation — the graph dual to the Voronoi boundaries - of randomly distributed points are treated as waveguides. The result is both efficient and accurate. They further describe how a moving listener may be modelled using a Voronoi tessellation of the same set of sites.

\section{Building the Apollonius diagram; point patterns, shapes and animation}

When building a diagram from music, we first have to identify what we wish to map to a site, before deciding how. That is, we have to identify the musical atoms we are going to map from.

This paper defines a musical atom as a note. A note may be pitched and/or timbral in quality, but always has a single Onset and Duration. Further, this paper compares distance between notes as their Inter-Onset Interval (IOI), that is a measurement of time between their onsets.

Time We may or may not use an axis to represent time. In one of our experiments, we pick an axis for time and model dynamics through a sweepline algorithm (Fortune's; §4.1).

Rather than using an explicit dimension (axis) to model time effects, we can embed time in the representation by designing dynamic diagrams evolving through time with a "cinematic" quality, which has the advantage of "freeing-up" one dimension, which can 
then be used to represent an additional note parameter or feature.

Note parameters We may choose from many possible properties of a note. For example those from the MIDI standard [6] such as volume, pitch, stereo (or multichannel) panning and modulation, or other synthesis parameters such as cutoff, resonance, distortion, waveshaping, chorus and so on.

\subsection{Voronoi assignment model (VAM)}

There is a great body of knowledge surrounding Voronoi diagrams, but little applied in the field of music. Our greatest problem is how to map music, in particular live music, to form sites within a Voronoi diagram. Taking the archetypal example of a 2D Euclidean diagram, we have two axes to somehow map the many possible dimensions of a musical event to.

We must map between a musical space and a Voronoi space, a process called Voronoi assignment. Okabe et al. [8, p.454] define four assumptions as the Voronoi assignment model which we summarise:

VAM1 Each point is located simultaneously before any assignment procedure occurs

VAM2 Each point remains fixed throughout the assignment process

VAM3 Each point is of equal importance

VAM4 This assumption describes the Voronoi model and is not reproduced here.

With live music, VAM1 and VAM2 present a problem for us, as we want to construct a diagram live, i.e., while the music is played or generated. We see one possible way around this problem in Section 4.1. VAM3 also presents a problem, since every musical sound is not of equal importance, to consider them as such is a simplification. Whether such a simplification is acceptable depends on the context, but we introduce a system for constructing a Voronoi diagram with individually weighted sites in Section 4.2.

\subsection{Visualising Relationships Between Notes}

We consider the following hypothesis: a basic perceptual structural piece of information is provided by neighborhood relations amongst "perceptual events." In particular, the Gestalt theory of perceptual organisation is based on such a notion. This is of particular interest to us as this theory of perception applies to auditory as well as visual stimuli (and possibly other senses, such as touch). Gestalt theory is based on a number of "principles" which are used to predict observed perceptual phenomena. Principles such as "proximity, similarity, closure, area, symmetry" can be based upon a notion of neighborhood relations.

One possible powerful computational framework to implement neighborhood relations which can support Gestalt theory is the medial axis or symmetry transform of Harry Blum (from pattern analysis), also known as the Voronoi diagram in computational geometry [5].

Symmetry Structures: Relations by Pairings The most immediate way to build relationships between events is to expand their surrounding "zone of influence" until this zone is in conflict with another, possibly many other, zone(s) expanded from other event(s), where an event can be a visual element (e.g., a point or edge from an object contour) or a note.

When the rate of expansion is uniform and homogeneous, each zone of influence represents the points closest to the event, and each frontier represents the limit of the influence of events by pairs. The full diagram thus built is a simulation of a geometric wave propagation through space and is equivalently referred to as the Medial Axis Transform of the Voronoi Diagram. The "frontiers" take in 2D the form of a graph, called Medial Axis or Voronoi Graph. Typically the rate of expansion mimics the Euclidean metric, but other, non-Euclidean (but uniform), metrics are also used in practice (e.g., the Manhattan, chessboard or chamfer metrics).

Once such a representation is built, all nearestneighbor relationships are readily available. In particular, the graph dual to the Medial Axis or Voronoi Graph, called the Delaunay Tessellation, is used to show such relationships between the original events. In $2 \mathrm{D}$, and for point events, it takes the form of a triangulation (and in 3D, for points, it connects these by tetrahedra).

The advantages of using such a definition for visualising events are multiple. First, immediate symmetries (by pairing) are made explicit as (Voronoi or Medial Axis) frontiers. Second, higher order structures can also be retrieved: (i) triangulations and convex hulls, via the Delaunay duals, (ii) hierarchical segmentation into regions, by grouping, (iii) simplification by structural smoothing (including the detection of outliers, small features, noise).

Still, other generalisations of such symmetry structures are available and useful in the dynamic context of representing events with time variations. In particular we consider below the extension to the weighted 


\section{Figure 2. Frames from an Apollonius diagram animation showing a sequence of five notes emerging radially from a central origin.}

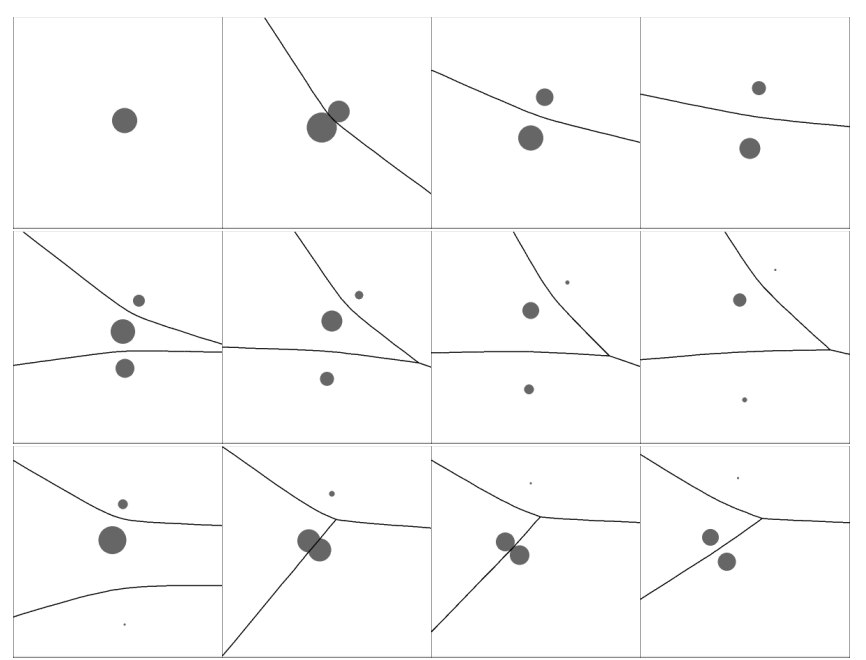

Voronoi diagram, where each event has a particular initial weight, which can then vary through time (e.g., a changing volume of a note). For point data, this is equivalent to initially replacing a point by a disk such that its radius reflect the weight. The resulting diagram and symmetry structure are also known as the Apollonius diagram and graph.

Apollonius diagrams Apollonius diagrams are a class of Voronoi diagrams where events are discs. The discs may have different areas and may overlap. We can view an Apollonius diagram as consisting of point events with differing weightings, the radius being proportional to its weighting. Figure 1 shows how the symmetry structure (or Voronoi edges) may be curved for such a diagram.

Weighting allows us to map dynamically an extra dimension beyond the number of axes of our diagram. A good choice for this mapping could be the volume of a note, or some other property which we perceive as bearing of one note over another, such the note's metrical level within a rhythm. Figure 2 shows a simple use of this representation for the visualisation of five notes.

\subsection{Behaviours}

In the previous section we saw how weighting can allow us to map an extra dimension beyond the spatial dimensions of a diagram. If we consider a diagram to be of a snapshot of time, then we can map many further

\section{Figure 3. Apollonius diagram animation} showing a "glissandi."

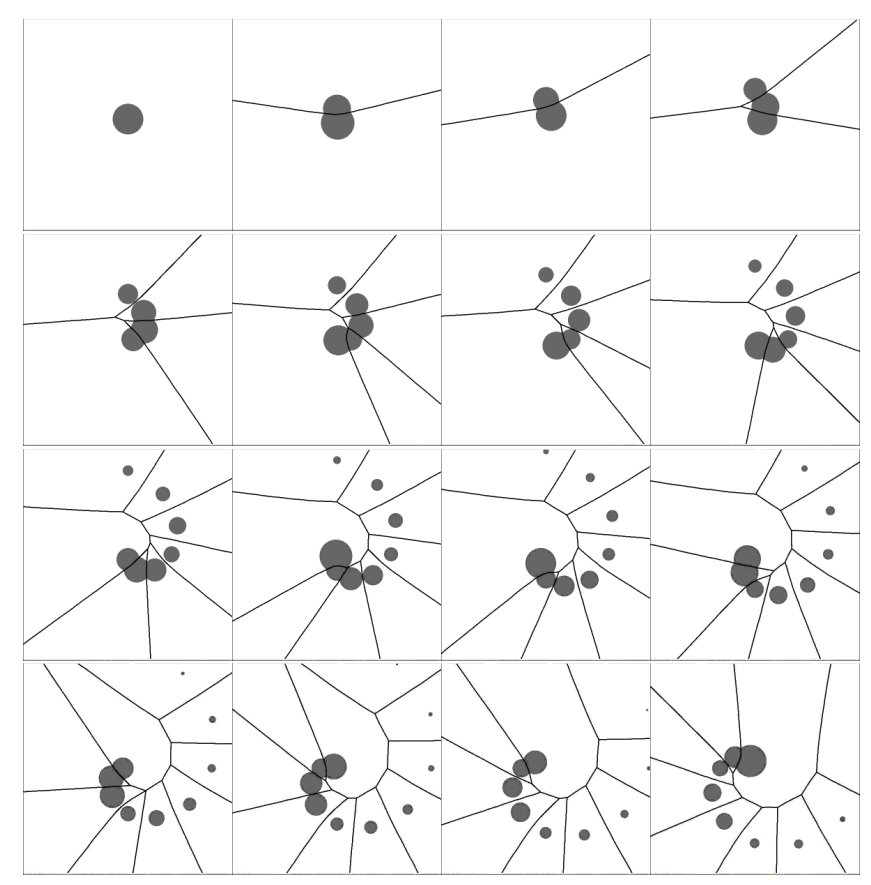

dimensions onto "how the sites move over time."

Firstly we consider the direction in which the event moves over time. For tonal music, we propose mapping a note's pitch to a direction. Taking the twelve tone scale commonly used within western classical music, we assign the twelve notes to the direction of the hours on a clock. For example, "glissandi" result in spiral evolutions as seen in Figure 3.

Here middle $\mathrm{C}$ travels in the same direction (straight up) as the $\mathrm{C}$ in the octaves above and below it. To differentiate between one octave and another, we propose to use speed of travel in the given direction so that a note of a higher octave travels at a higher speed.

So far we have described visualising a note as a site moving in a particular direction over time. However, this movement does not have to be linear. For example, we may visualise a note as not only moving in a particular direction from the origin, but in addition oscillating from side to side as it travels. For example, we may use a sinusoid with amplitude and frequency mapped from vibrato, distortion, reverberation, or even raw sound samples themselves. ${ }^{1}$

\footnotetext{
${ }^{1}$ Vibrato: a musical effect, produced in singing and on musical instruments by a regular pulsating change of pitch, which is used to add expression and vocal-like qualities to instrumental music. Distortion: any kind of deformation of a waveform. Reverberation: persistence of sound in a particular space after the original sound is removed. [From Wikipedia].
} 
(a)

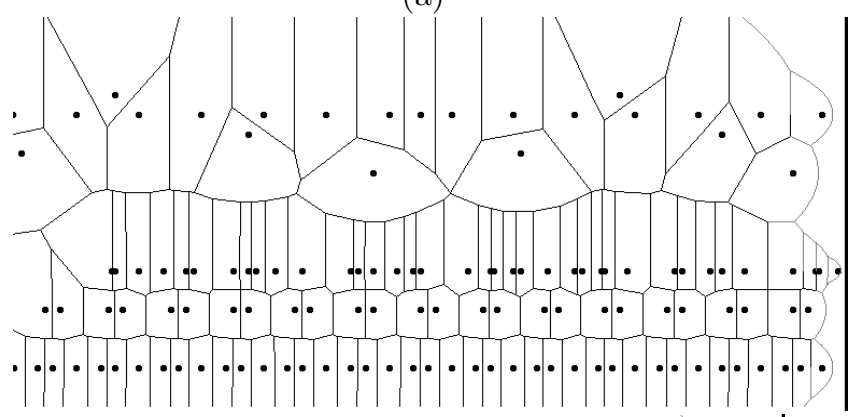

(b)

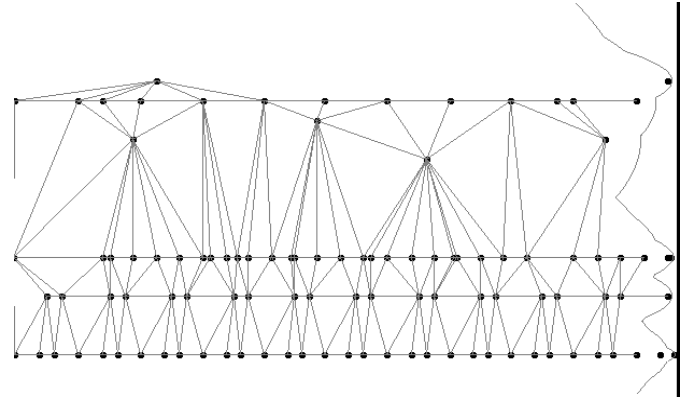

Figure 4. Sweepline, showing (a) Voronoi diagram and (b) Delaunay triangulation. $X$ axis is time, and $Y$ axis is instrument.

\section{Visualisation}

On-line rendering of live performance Here we visualise the on-line construction of a Voronoi diagram towards two different ends. One is as a reference for the musicians, so they may get a visualisation of the music they are producing. This use deserves experimentation but if we assume that musicians should place all their focus on the sounds they're producing, it would follow that such a visualisation would be too distracting to be useful.

The second proposed use is as a reference for audience members. Again, we could say that the audience should be focussing on the music itself, but let us consider the increasingly common scenario of the laptop performance. Here a performer gazes into their computer laptop screen taking actions unseen to the audience, appearing to be motionless and sometimes disinterested, in a way incongruous to the often highly dynamic sound produced. This perceived distance between the performer and their performance can also be reflected in the audiences sense of involvement and therefore immersion in the performance. Here an online Voronoi diagram projected behind or even onto the performer, representing some aspects behind the structure of the music might lend some visual dynamism to the performance.

\subsection{Representing time along a sweepline}

Sweep algorithms are useful for solving many geometrical problems, where a plane sweeps across a space, from left to right, calculating values across the plane as it goes. This is akin to calculating a series of n-1 dimensional spaces in order to solve an $\mathrm{n}$ dimensional space and can be an efficient as well as easy to understand approach. However a sweep line can only calculate values in response to events across its plane and to the left of it. This makes things difficult when using a sweepline to find Voronoi vertices, we can't calculate vertices only based on sites already seen, as sites found during later iterations are likely to interfere with them.

Steve Fortune found [2] that while vertices cannot be calculated along the sweep-line, they can be calculated with certainty halfway between the sweep-line and the closest sites. These points of certainty between a site and the sweep-line forms a parabola, and where the parabolas of two closest sites touch, we know a Voronoi edge crosses. Where this crossing lies at the centre of a circle with its edges touching its sites and also the sweep-line, we know the crossing is a Voronoi vertex.

Fortune's sweep-line is both elegant and efficient, but there is another reason for highlighting it here. It allows us to ignore VAM1 as described in section 3.1. If one of the Voronoi axes is time, we can place the sweep-line on this axis and have it progress with the movement of time. This allows us what is termed online construction of a Voronoi diagram of live music (Fig. 4).

In order to explore this use further, we adapted some software by Odgaard and Nielsen so that it could be controlled by live music generation software. When a musical event occurs, it is represented as a Voronoi site at the sweep-line, shown on the far right of the diagram. The site's position on the $\mathrm{Y}$ axis is chosen according to the instrument and pitch. The regular events shown at the bottom of the diagram represent a simple rhythm, and the clustering patterns above represent a melodic structure. Several features related to the Voronoi diagram can be displayed including sites, Voronoi edges, the beachline, Delaunay edges and Delaunay circles. The Voronoi diagram is incrementally calculated at a high framerate allowing smooth transitions, particularly evident in the beachline.

This software was trialled during a performance by Alex McLean and Dave Griffiths at the Make Art festival in Poitiers, France, 2006. The results were visually pleasing and later feedback from audience members was encouraging. 


\section{Figure 5. Apollonius diagram animation showing a polyphonic sequence of notes.}

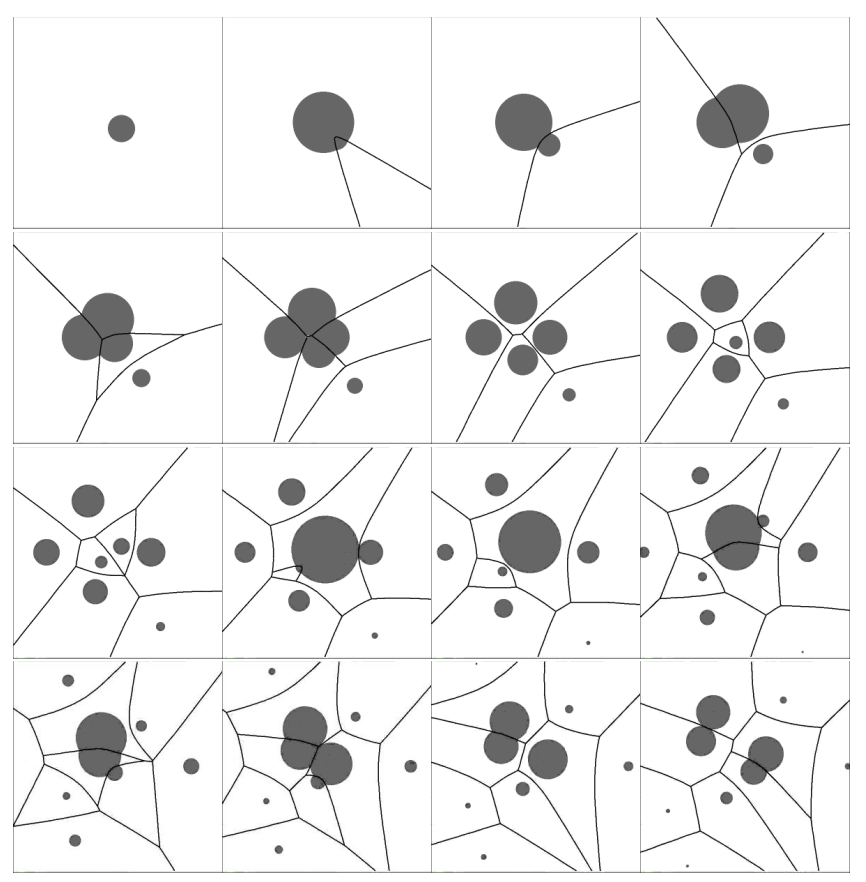

\subsection{Animation of an Apollonius Graph}

Animation allows us to represents snapshots of time as video frames. This frees one dimension, allowing us to place a site anywhere in the $2 \mathrm{D}$ plane, not just along the sweepline as in the software described in the previous section. Within a $2 \mathrm{D}$ plane it is possible to define a rich action space, within which sites do not have fixed positions, but behaviours described in Section 3.3. These behaviours may have parameters beyond the number of dimensions of the action space that they are contained within.

To demonstrate the usefulness of this, we produced software that takes MIDI [6] or Open Sound Control [9] input to place Voronoi sites within a two dimensional display. The sites have weightings, the result being an Apollonius graph as described in section 3.2 (Fig. 5).

Each site is placed with the following parameters:

startX Origin along the $\mathrm{X}$ axis.

startY Origin along the Y axis.

weight Starting weight of the site, represented as a disc with a size proportional to this weight.

duration The time in frames that the site should persist. The weight of the site is decreased linearly towards zero over this duration. speed

The speed in pixels at which the site travels from its origin.

direction The direction in radians at which the site travels from its origin.

modulation Amount of modulation or 'wobble' applied to the path of the site.

More details, examples, video animations and source code, are available from: doc.gold.ac.uk/soundvis/

\section{References}

[1] R. Bencina. The metasurface: applying natural neighbour interpolation to two-to-many mapping. In NIME '05: Proceedings of the 2005 conference on New interfaces for musical expression, pages 101-104. National University of Singapore, 2004.

[2] S. Fortune. A sweepline algorithm for Voronoi diagrams. In $S C G$ '86: Proceedings of the second annual symposium on Computational geometry, pages 313-322, New York, NY, USA, 1986. ACM Press.

[3] M. Hamanaka and K. Hirata. Applying Voronoi diagrams in the automatic grouping of polyphony. Information Technology Letters, 1(1):101-102, September 2002.

[4] F. Lerdahl and R. Jackendoff. A Generative Theory of Tonal Music. MIT Press, Cambridge, MA, 1983.

[5] F. F. Leymarie. Aesthetic computing and shape. Aesthetic Computing, Paul Fishwick, ed., chapter 14. MIT Press, April 2006.

[6] MIDI Manufacturers Association. The Complete MIDI 1.0 Detailed Specification, v96.1 edition, 2001. www.midi.org.

[7] B. D. Moor, A. Nackaerts, and B. Schiettecatte. Realtime acoustics simulation using mesh-tracing. In Proceedings of the International Computer Music Conference 2003, 2003.

[8] A. Okabe, B. Boots, K. Sugihara, and S. N. Chiu. Spatial Tessellations: Concepts and Applications of Voronoi Diagrams. Series in Probability and Statistics. John Wiley and Sons, Inc., 2nd ed. edition, 2000.

[9] M. Wright. OpenSound Control Specification. Center for New Music and Audio Technologies, UC Berkeley, 2002. 